\title{
Peripheral Lymphocyte Multidrug Resistance Activity as a Predictive Tool of Biological Therapeutic Response in Rheumatoid Arthritis
}

\author{
Gergely Toldi (iD, Patrizia Batel, Sándor Baráth, Péter Szerémy, András Apjok, Kata Filkor, \\ Sándor Szántó, Gabriella Szúcs (D), Szilvia Szamosi, Thomas Häupl, Andreas Grützkau, \\ and Zoltán Szekanecz
}

\begin{abstract}
Objective. Multidrug resistance (MDR) transporters may be used as biomarkers to monitor disease progression in RA and as a predictive tool to establish responsiveness to biological therapy. In this multicenter clinical trial, we aimed to assess the predictive value of activity measurement of transporters MDR1, MD resistance protein (MRP)1, and breast cancer resistance protein (BCRP) for biological therapeutic response in RA before the initiation of biological therapy as well as 4 to 6 and 12 weeks after.

Methods. Peripheral blood samples were collected from 27 responders and 12 nonresponders to biological disease-modifying antirheumatic drugs (bDMARD) at the indicated timepoints as well as from 35 healthy controls. MDR activity factor (MAF) of MDR1, MRP1, and BCRP was measured in CD3+ and CD19+ cells using the Solvo MDQ Kit and cell surface staining by flow cytometry following peripheral blood mononuclear cells isolation.

Results. At the start of therapy, MAFC (composite MAF of MRP1 and MDR1) and MAFMDR values, and at 4 to 6 weeks of treatment, MAFC, MAFMRP, and MAFMDR values of CD3 cells were higher in nonresponders compared to responders. Receiver-operation characteristic curve analysis revealed that RA patients with MAFC values above 21.3 in CD3 cells at the start of bDMARD therapy are likely to be nonresponders. At 4 to 6 weeks of treatment, these also predict unfavorable response: MAFC values above 20.3, MAFMRP values above 6.0, and MAFMDR values above 13.9 in CD3 cells.

Conclusion. Our results indicate that the determination of MAFC values in CD3 cells of patients with RA may be of predictive value prior to the initiation of biological therapy, to establish whether the patient will demonstrate sufficient therapeutic response. (First Release March 1 2019; J Rheumatol 2019;46:572-8; doi:10.3899/jrheum.180793)
\end{abstract}

Key Indexing Terms:

MULTIDRUG RESISTANCE

THERAPEUTIC RESPONSE

T CELLS

RHEUMATOID ARTHRITIS

\footnotetext{
From the MDQuest Ltd., Szeged, Hungary; German Rheumatism Research Centre Berlin (DRFZ), an Institute of the Leibniz Association, Berlin, Germany; Department of Laboratory Medicine, University of Debrecen, Third Department of Internal Medicine, University of Debrecen, Debrecen, Hungary.

KF is employed by MDQuest Ltd.

G. Toldi, MD, PhD, MDQuest Ltd.; P. Batel, MSc, DRFZ; S. Baráth, MD, $P h D$, Department of Laboratory Medicine, University of Debrecen; P. Szerémy, MSc, MDQuest Ltd.; A. Apjok, MD, MDQuest Ltd.; K. Filkor, PhD, MDQuest Ltd.; S. Szántó, MD, DSc, Third Department of Internal Medicine, University of Debrecen; G. Szücs, MD, DSc, Third Department of Internal Medicine, University of Debrecen; S. Szamosi, $M D, P h D$, Third Department of Internal Medicine, University of Debrecen; T. Häupl, MD, PhD, DRFZ; A. Grützkau, PhD, DRFZ; Z. Szekanecz, MD, DSc, Third Department of Internal Medicine, University of Debrecen.

Address correspondence to Dr. G. Toldi, MDQuest Ltd., Közép fasor 52, Szeged,H-6726, Hungary.E-mail: toldi@mdquest.hu

Accepted for publication November 8, 2018.
}

Rheumatoid arthritis (RA) affects about $0.5-1 \%$ of the population and causes chronic synovial inflammation, eventually leading to joint destruction and disability ${ }^{1}$. Early diagnosis and immediate, effective therapy are crucial to prevent joint deterioration, functional disability, and unfavorable disease outcome. The optimal management of RA is needed within 3-6 months after the onset of disease; therefore, a very narrow window of opportunity is present to achieve remission or at least low disease activity ${ }^{2,3}$. It is very important to predict the efficacy of expensive biologicals at early stages of treatment. Although a new generation of drugs is available, there are no validated circulating biomarkers to use prognostically or to predict response to specific therapies ${ }^{4}$.

Multidrug resistance (MDR) ATP-binding cassette (ABC)

Personal non-commercial use only. The Journal of Rheumatology Copyright $\subset$ $\subset$ 2019. All rights reserved 
transporters [MDR 1/P-gp/ABCB1, MD resistance protein (MRP)1/ABCC1, breast cancer resistance protein (BCRP)/ABCG2] are important components in the development of drug resistance in malignancies ${ }^{5}$ and in autoimmune conditions, such as $\mathrm{RA}^{6}$. Although studies of the crystal structure and function of MDR-ABC transporters suggest that they are not directly involved in the release of cytokines and chemokines, they may extrude other intracellular small molecules, thus influencing the inflammatory balance. They may play an important role in the pathogenesis of RA by influencing cell migration, proliferation, and inflammation in an indirect manner. Therefore, MDR-ABC transporters may also be important biomarkers of disease progression in RA. The assessment of MDR protein activity may help physicians to evaluate how patients will respond to biological treatment and may support the decision about whether it is necessary to modify treatment.

The most important conventional synthetic disease-modifying antirheumatic drugs [csDMARD; including methotrexate (MTX), sulfasalazine, leflunomide, and hydroxychloroquine] are substrates of MDR proteins. For this reason, MDR activity of patients with RA receiving csDMARD therapy has been extensively studied, and the expression, polymorphisms, and activity of MDR proteins have been linked to the therapeutic success of csDMARD, especially that of MTX. However, little is known about the relation of MDR proteins to therapeutic success of biologicals, such as anti-tumor necrosis factor (TNF) agents. Although these molecules do not enter the cell, and are therefore not substrates of MDR proteins, the endobiotics, such as the cytokines they target, are known to interact with these transporters ${ }^{7,8,9}$. Through influencing the distribution of cytokines and other soluble factors within the cell and in its most proximal environment, the function of $\mathrm{T}$ and $\mathrm{B}$ cells may be affected by the activity of the transporters, balancing the effect of such factors on lymphocyte activation, proliferation, production of other cytokines and antibodies, and so on. Therefore, MDR activity may be used as a biomarker of therapeutic success in RA and other autoimmune disorders.

In this multicenter clinical trial, we aimed to assess the predictive value of flow cytometry-based multidrug resistance activity measurement for biological therapeutic response in RA. We aimed to assess the activity of 3 clinically relevant MDR proteins (MDR1, MRP1, BCRP) in CD3+ and CD19+ lymphocytes of patients with RA before as well as 4 to 6 and 12 weeks after the initiation of biological therapy.

\section{MATERIALS AND METHODS}

Patient recruitment. At the outpatient clinics of the Department of Rheumatology, University of Debrecen, Hungary, and the Department of Rheumatology and Clinical Immunology, Charité, Berlin, Germany, 39 patients with RA were recruited. Patients were sampled before the start of biological treatment as well as between 4 and 6 weeks and at 12 weeks of treatment. The 28-joint count Disease Activity Score (DAS28) and C-reactive protein (CRP) values were also recorded at this time. Patients were regarded as nonresponders $(\mathrm{n}=12)$ if DAS28 values showed a decrease of $<25 \%$ between the start of biologicals and at 12 weeks of treatment (arbitrary cutoff). Patient characteristics as well as details of the therapy received are included in Table 1.

Healthy controls $(\mathrm{n}=35)$ were sampled at the Department of Rheumatology, University of Debrecen, Hungary, on a single occasion. They had no history of autoimmune disorders including RA and a negative status upon physical examination as well as no infectious symptoms within 3 weeks before sampling.

Exclusion criteria for all participants included chronic infectious diseases requiring systemic treatment, autoimmune diseases other than RA, immunodeficiencies, allergic diseases, and hematological malignancies or solid tumors.

Written informed consent was obtained from all participants and the study adhered to the tenets of the most recent revision of the Declaration of Helsinki. Ethical approval for the study was granted by local ethics committees of the University of Debrecen (TUKEB 21018/2014/EKU) and Charité (EA1/193/10), respectively.

Peripheral blood mononuclear cell (PBMC) isolation. EDTA anticoagulated peripheral blood samples were collected $(6 \mathrm{ml})$. PBMC were separated by density gradient centrifugation using Ficoll Histopaque-1077 (Sigma-Aldrich) according to the manufacturer's instructions.

Flow cytometry. Measurements were conducted on a BD FACSCalibur flow cytometer (BD Biosciences) equipped with $488 \mathrm{~nm}$ and $635 \mathrm{~nm}$ lasers or on a Miltenyi MACSQuant flow cytometer, equipped with $405 \mathrm{~nm}, 488 \mathrm{~nm}$, and $638 \mathrm{~nm}$ lasers.

The Solvo MDQ Kit was used strictly following the manufacturer's instructions. In this assay, fluorescent reporter substrates are trapped in the cytoplasm and pumped out by MDR proteins depending on the presence or absence of highly selective inhibitors, allowing for quantitative, standardized assessment. PBMC were loaded with fluorescent MDR activity reporter substrates (1.25 uM working solution of Calcein-AM for MDR1 and MRP1, emission (em): $515 \mathrm{~nm}$, incubated for exactly 10 min followed by rapid centrifugation and $1 \mathrm{mM}$ working solution of mitoxantrone for BCRP, $\mathrm{em}$ : $684 \mathrm{~nm}$, incubated for exactly $30 \mathrm{~min}$ followed by rapid centrifugation) and treated with highly selective MDR protein inhibitors (12.7 mM working solution of verapamil for MDR1 and MRP1, $2 \mathrm{mM}$ working solution of indomethacin for MRP1, and $0.1 \mathrm{mM}$ working solution of KO134 for $\mathrm{BCRP}$ ) to obtain multidrug activity factor (MAF) values (Figure 1). Note that the above working concentrations are further diluted upon addition to the cell suspension to avoid cell toxicity as described in the manufacturer's instructions.

Cell surface staining was applied to select CD3+ and CD19+ cells using antihuman CD3-PerCP and CD19-PE monoclonal antibodies (BD Biosciences) in the case of calcein-AM-stained cells and antihuman CD3-FITC and CD19-PE monoclonal antibodies (BD Biosciences) in the case of mitoxantrone-stained cells, according to the manufacturer's instructions. Samples were run on a flow cytometer immediately following cell surface staining.

Activities of multidrug transporters are reflected by the difference between the amount of calcein/mitoxantrone accumulated in the presence or absence of the selective inhibitor(s). When calculating the MAF values, this accumulation difference is normalized to the dye uptake measured in the presence of the inhibitor. Thus, the result of the test becomes independent from factors influencing the cellular accumulation of calcein/mitoxantrone other than the activity of the multidrug transporters. The interassay variability of the test is coefficient of variation $<10 \%$.

MAF values were calculated from the difference between the geometric mean fluorescent intensity of cells with and without the highly selective inhibitors. Calculations followed these formulas:

MAFC (composite MAF of MRP1 and MDR1) $=100 \times($ Fmax - Fo $) /$ Fmax MAF of MRP1 $=100 \times($ FMRP $1-$ Fo $) /$ Fmax

MAF of MDR $1=$ MAFC - MAF of MRP1

MAF of BCRP $=100 \times($ FMX - Fo $) /$ FMX

Fmax/FMX: Calcein/mitoxantrone fluorescence with verapamil or KO134

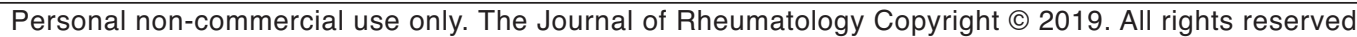


Table 1. Clinical characteristics of responder and nonresponder patients with RA as well as healthy controls.

\begin{tabular}{lccc}
\hline Characteristics & $\begin{array}{c}\text { Healthy Controls, } \\
\mathrm{n}=35\end{array}$ & $\begin{array}{c}\text { Responders, } \\
\mathrm{n}=27\end{array}$ & $\begin{array}{c}\text { Nonresponders, } \\
\mathrm{n}=12\end{array}$ \\
\hline Age, yrs & $54(42-62)$ & $56(49-61)$ & $51(39-61)$ \\
Sex (male/female) & $4 / 31$ & $2 / 25$ & $1 / 11$ \\
RA duration, yrs & & $10(5-14)$ & $8.5(5-15)$ \\
No. patients receiving MTX & $15(56)$ & $6(50)$ \\
No. patients receiving prednisolone & $9(33)$ & $5(42)$ \\
No. patients receiving adalimumab & $2(7)$ & $1(8)$ \\
No. patients receiving certolizumab pegol & & $5(19)$ & $3(25)$ \\
No. patients receiving etanercept & $7(26)$ & $3(25)$ \\
No. patients receiving abatacept & & $13(48)$ & $5(42)$
\end{tabular}

Data are expressed as median (IQR) for continuous variables and as $\mathrm{n}(\%)$ for categorical variables. RA: rheumatoid arthritis; MTX: methotrexate.
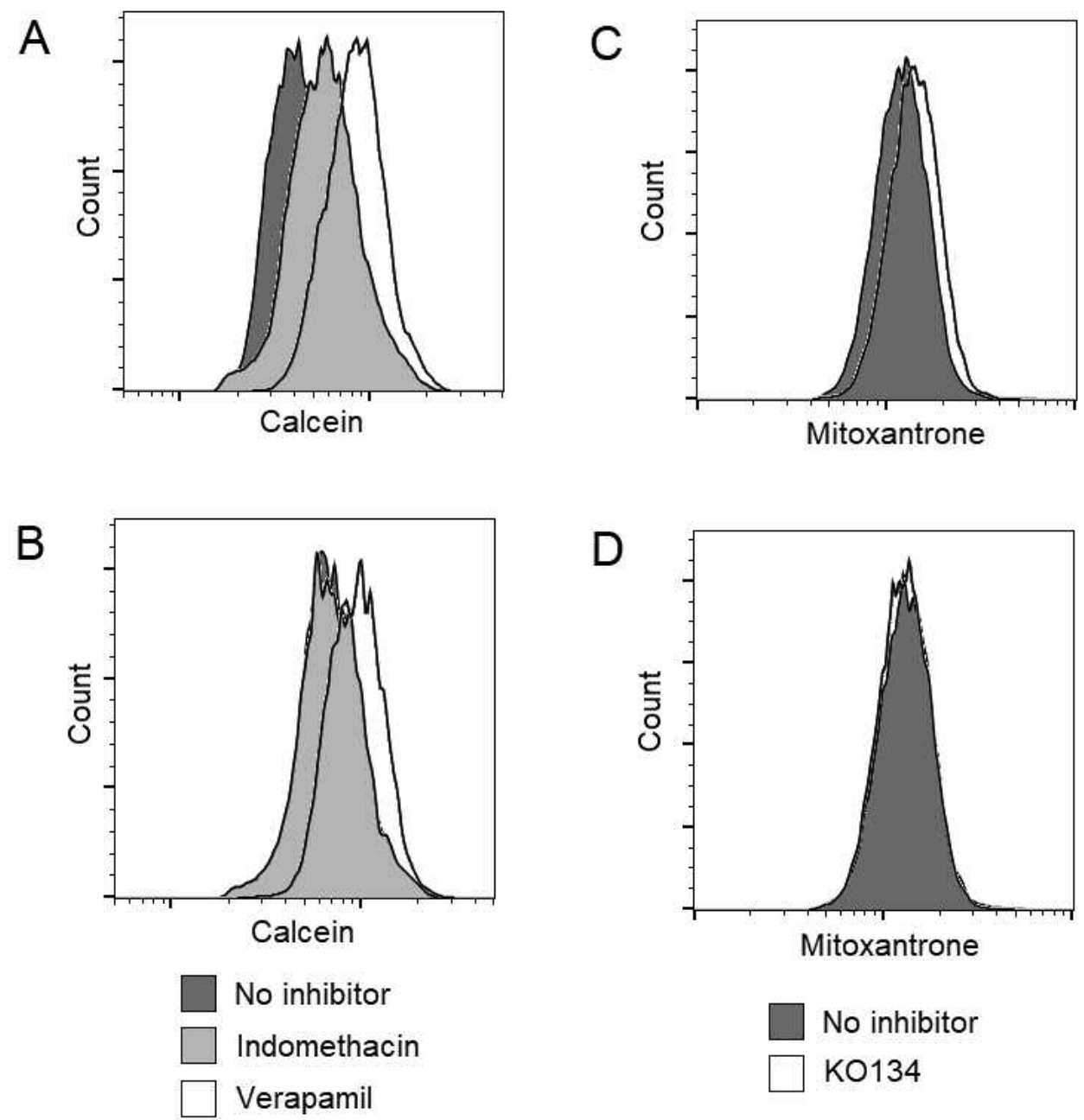

Figure 1. The effects of highly selective inhibitors of MRP1 (indomethacin) and MRP1 + MDR1 (verapamil) on calcein substrate fluorescence intensity at high (A) and low (B) transporter activity, and the effects of the highly selective inhibitor of BCRP (KO134) on mitoxantrone substrate fluorescence intensity at high (C) and low (D) transporter activity in CD3 cells. Data from representative samples of patients with rheumatoid arthritis. MDR1: multidrug resistance transporter; MRP1: MDR protein 1; BCRP: breast cancer resistance protein.

In the formulas, Fo means fluorescence without inhibitor, and FMRP1 means calcein fluorescence with indomethacin.
Statistics. Comparisons were made using the Kruskal-Wallis test or the Mann-Whitney $U$ test because the distribution of data appeared to be 
non-normal according to the Shapiro-Wilk test. $\mathrm{P}$ values $<0.05$ were considered significant. Statistics were calculated using the GraphPad Prism 5 software.

\section{RESULTS}

DAS28 values decreased upon treatment in responders in contrast to nonresponders. Of note, initial DAS28 values were higher in the responder group compared to nonresponders (Table 2, Figure 2). No differences in CRP values or changes in CRP were observed in either group.

MAF of MRP1 in CD3 cells was higher at 12 weeks in nonresponders compared to controls. No other statistically significant difference was noted in MAF values between controls and patients with RA.

Control values were within the reference range established in our earlier study ${ }^{10}$.

At the start of therapy, MAFC and MAFMDR values of CD3 cells were higher in nonresponders compared to responders. At 6 weeks, MAFC, MAFMRP, and MAFMDR values of CD3 cells as well as MAFMRP values of CD19 cells were higher in nonresponders compared to responders (Table 2).

No significant changes were demonstrated in MAF values in the respective RA patient groups with the progress of treatment (Figure 3).

No difference was demonstrated in MAFBCRP values in CD3 or CD19 cells between responders and nonresponders.

Receiver-operation characteristic (ROC) curve analysis was performed to evaluate the predictive value of MAF for response to treatment in patients with RA at the start of biological therapy and at 6 weeks. Cutoff thresholds were calculated for MAF values with ROC of adequate $\mathrm{p}$ and area under the curve (AUC) values (Figure 4). Patients with MAF values above the respective cutoff thresholds are likely to be nonresponders to treatment (MAFC of CD3 cells at 0 weeks: $\mathrm{p}=0.043$, AUC $=0.68$; MAFC of CD3 cells at 6 weeks: $\mathrm{p}=0.033, \mathrm{AUC}=0.72$; MAFMDR on $\mathrm{CD} 3$ cells at 6 weeks: $\mathrm{p}=0.048, \mathrm{AUC}=0.70 ;$ MAFMRP on $\mathrm{CD} 3$ cells at 6 weeks: $\mathrm{p}=0.049, \mathrm{AUC}=0.69$ ).

\section{DISCUSSION}

Our results indicate that at the start of therapy, MAFC and MAFMDR values, and later at 4 to 6 weeks of treatment, MAFC, MAFMRP and MAFMDR values of CD3 cells were higher in nonresponders to biological DMARD (bDMARD) compared to responders among patients with RA. ROC analysis revealed that RA patients with MAFC values $>21.3$ in CD3 cells at the start of bDMARD therapy are likely to be nonresponders.

While MDR1 expression on healthy CD4+ and CD19+ lymphocytes is only marginal, significant upregulation was demonstrated in patients with $\mathrm{RA}^{11,12}$ as a result of the presence of damage-associated molecular patterns. Further, the expression level of MDR1 was significantly elevated in MTX nonresponder patients compared to responders. The YB-1 transcriptional factor may have an essential role in the regulation of MDR1 in lymphocytes of patients with RA by translocation from the cytoplasm into the nucleus. Inflammation-derived TNF- $\alpha$ appears to play a crucial role in this phenomenon $^{13}$.

In an earlier study, rhodamine 123 was used as a substrate of MDR1 and verapamil as a transporter inhibitor ${ }^{14}$. Flow cytometry-based analysis did not find any correlations between MTX responders and nonresponders at baseline; however, in nonresponders the functional activity was upregulated 4 months following therapy.

In a more recent study, the activity of MDR1 and MRP1 was investigated on different leukocyte subsets: granulocytes, monocytes, lymphocytes, CD4+, CD8+, and CD19+ cells from patients and controls (traumatic injury patients and healthy volunteers, respectively $)^{15}$. Based on DAS28 scores, patients with RA fell into MTX responder and nonresponder groups. Because side effects easily develop during MTX treatment, an additional MTX intolerant group was generated with intolerable side effects. In the case of granulocytes, the functional activity of MRP1 was significantly higher in MTX responders versus MTX nonresponders. Further, even higher

Table 2. Activity of various MDR transporters on CD3 and CD19 cells in RA patients and healthy controls.

\begin{tabular}{|c|c|c|c|c|c|c|c|}
\hline \multirow[t]{2}{*}{ Variables } & \multirow[t]{2}{*}{ Controls } & \multicolumn{2}{|c|}{0 Week } & \multicolumn{2}{|c|}{6 Weeks } & \multicolumn{2}{|c|}{12 Weeks } \\
\hline & & Responders & Nonresponders & Responders & Nonresponders & Responders & Nonresponders \\
\hline DAS28 & - & $5.94(5.11-6.17)$ & $4.65^{\mathrm{b}}(3.33-5.23)$ & $3.71^{\mathrm{c}}(2.79-4.45)$ & $3.93(3.14-4.50)$ & $3.00^{\mathrm{c}}(2.23-3.67)$ & $3.90^{\mathrm{b}}(2.81-4.90)$ \\
\hline CD3 MAFC & $18.3(14.7-22.9)$ & $18.9(14.0-25.2)$ & $23.5^{\mathrm{b}}(17.1-33.7)$ & $17.1(12.3-22.6)$ & $22.7^{\mathrm{b}}(16.7-29.2)$ & $18.3(15.7-24.2)$ & $25.2(15.9-30.7)$ \\
\hline CD3 MAFMRP & $3.1(1.2-5.7)$ & $4.8(0.0-8.0)$ & $5.7(2.2-8.0)$ & $2.2(0.0-7.9)$ & $8.4^{\mathrm{b}}(2.1-11.3)$ & $5.7(3.7-8.5)$ & $7.7^{\mathrm{a}}(4.0-11.6)$ \\
\hline CD3 MAFMDR & $14.6(12.5-18.1)$ & $12.9(11.0-16.7)$ & $19.1^{\mathrm{b}}(11.2-24.0)$ & $12.4(11.2-15.4)$ & $15.8^{\mathrm{b}}(14.3-18.7)$ & $12.5(9.2-17.5)$ & $13.6(6.0-20.0)$ \\
\hline CD19 MAFMRP & $2.2(0.0-6.3)$ & $0.9(0.0-7.7)$ & $4.4(0.0-5.8)$ & $0.6(0.0-5.1)$ & $6.8^{\mathrm{b}}(0.5-9.6)$ & $3.2(0.3-6.8)$ & $5.1(1.9-10.9)$ \\
\hline CD19 MAFMDR & $9.9(8.0-14.0)$ & $11.1(6.0-16.3)$ & $15.7(8.4-25.4)$ & $11.4(5.3-14.8)$ & $13.6(8.6-17.7)$ & $14.0(7.1-17.7)$ & $8.8(1.9-15.7)$ \\
\hline CD19 MAFBCRP & $3.8(1.0-6.3)$ & $3.1(0.7-7.0)$ & $4.5(0.0-11.0)$ & $2.7(0.0-5.2)$ & $5.0(3.1-8.4)$ & $2.9(1.3-5.1)$ & $3.0(1.8-3.7)$ \\
\hline
\end{tabular}

Data are expressed as median (IQR). P < $0.05 .{ }^{a}$ versus control, ${ }^{\mathrm{b}}$ versus responder, ${ }^{\mathrm{c}}$ versus 0 -week value. MDR: multidrug resistance; RA: rheumatoid arthritis; DAS28: 28-joint count Disease Activity Score; CRP: C-reactive protein; MAFC: composite multidrug activity factor (of MRP1 and MDR1 activity); MAFMRP: multidrug activity factor of multidrug resistance protein 1; MAFMDR: MAF of multidrug resistance 1; MAFBCRP: MAF of breast cancer resistance protein.

Personal non-commercial use only. The Journal of Rheumatology Copyright (C) 2019. All rights reserved. 


\section{Responder}

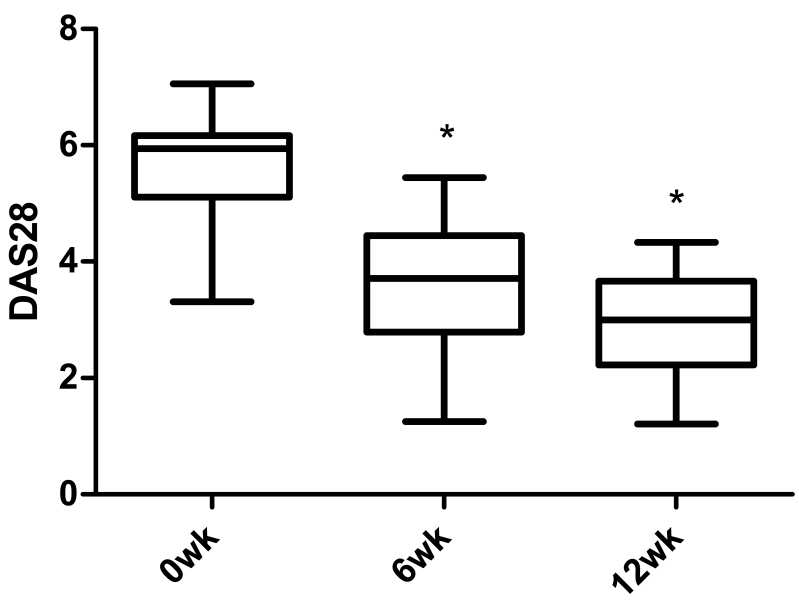

Non-responder

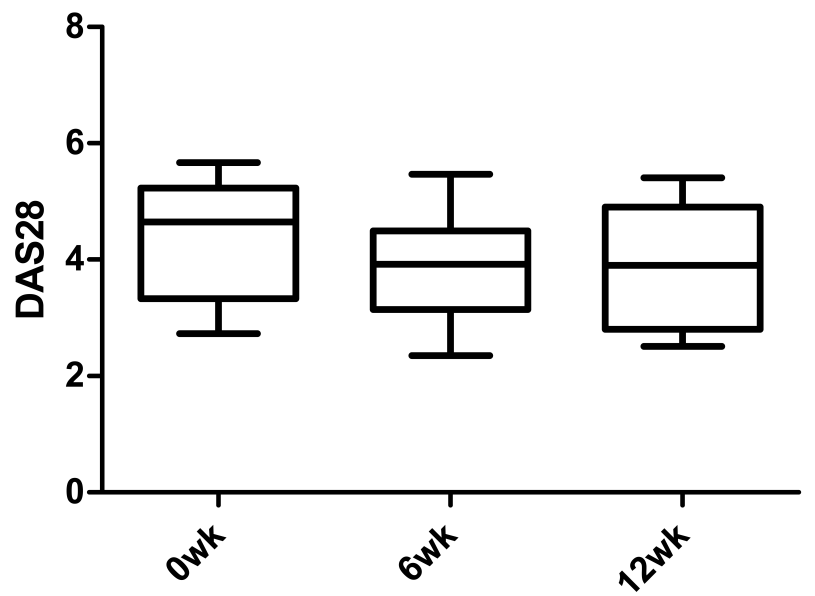

Figure 2. DAS28 values at different sampling timepoints in responder and nonresponder patients with rheumatoid arthritis. $* \mathrm{P}<0.05$ vs 0 weeks. DAS28: 28-joint count Disease Activity Score.

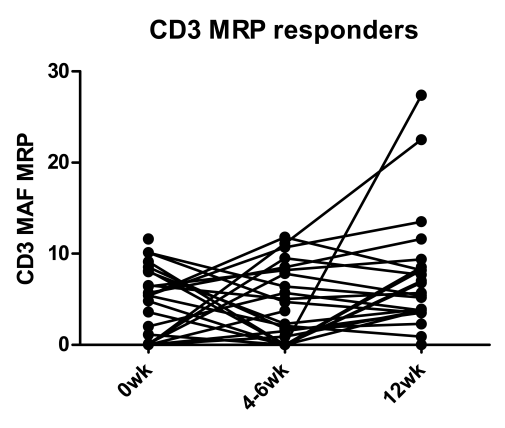

CD3 MRP non-responders

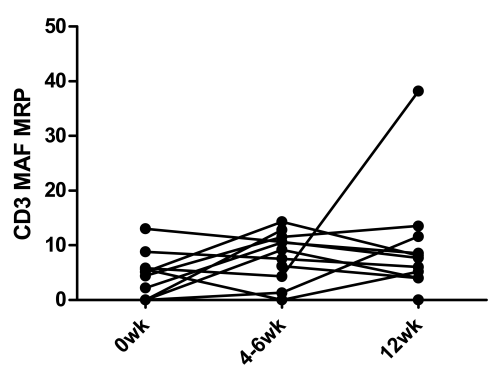

CD3 MDR responders

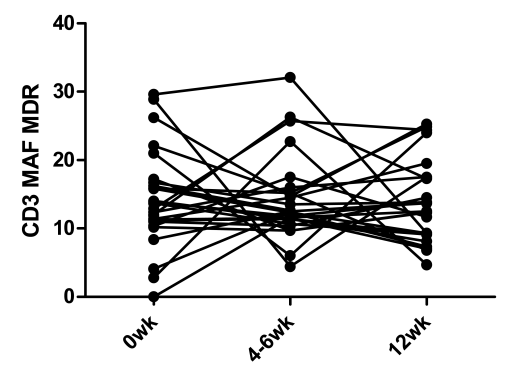

CD3 MDR non-responders

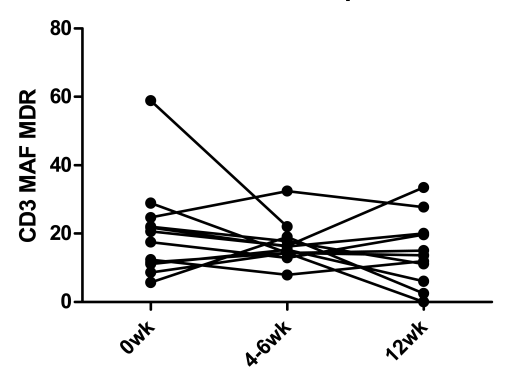

CD3 BCRP responders

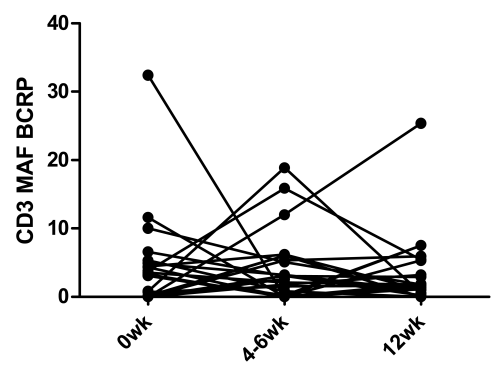

CD3 BCRP non-responders

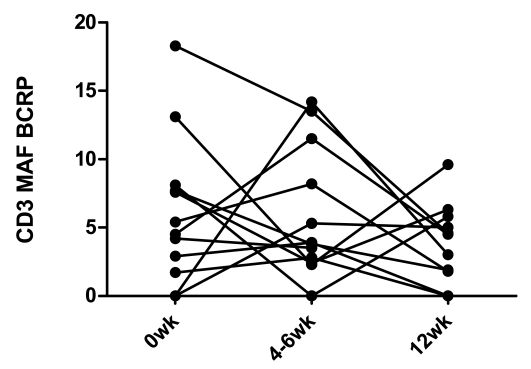

Figure 3. Individual changes of MAF values on CD3 cells over time in responder $(\mathrm{n}=27)$ and nonresponder $(\mathrm{n}=12)$ patients with rheumatoid arthritis $(\mathrm{RA})$ No significant changes were demonstrated in MAF values in the RA patient groups with the progress of treatment. MDR: multidrug resistance; MAF: MDR activity factor; MRP: MDR protein; BCRP: breast cancer resistance protein.

functional activity was demonstrated in MTX-intolerant individuals in comparison with MTX responders. Therefore, the authors concluded that determining MAF values might be useful in predicting MTX intolerance to avoid harmful side effects of MTX therapy ${ }^{15}$.
Although the role of MDR transporter activity in the prediction of response to MTX has been characterized in RA, little is known about the relationship of MDR proteins to therapeutic success of biologicals. In contrast to MTX and other csDMARD, these molecules do not enter the cell, and 
ROC of CD3 MAF C at Owk

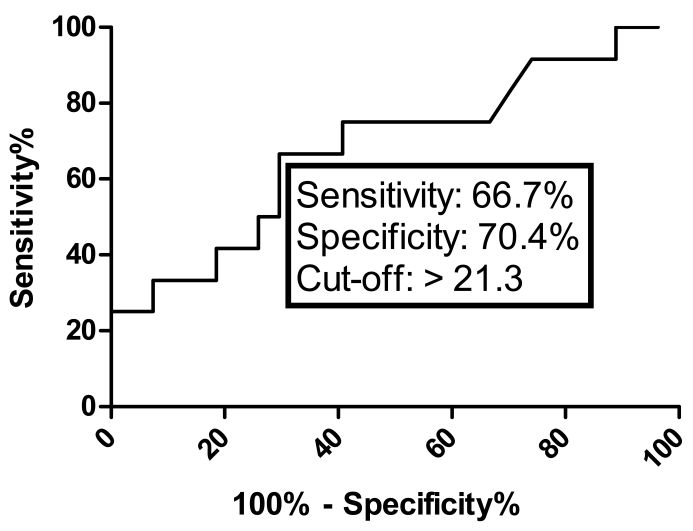

ROC of CD3 MAF MRP at 6wk

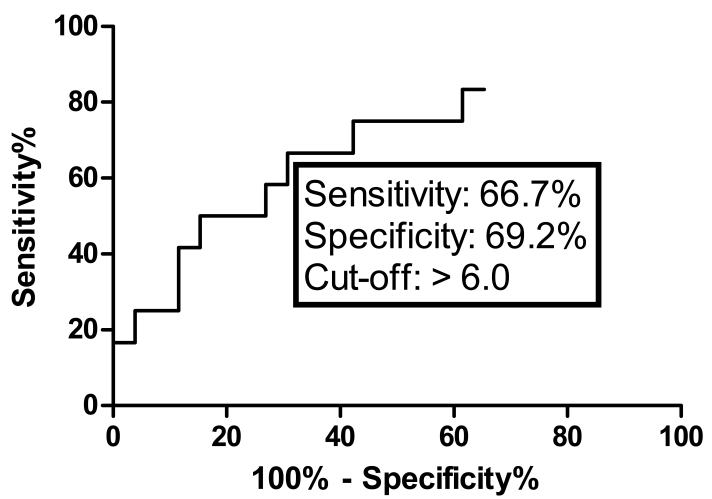

ROC of CD3 MAF C at 6wk

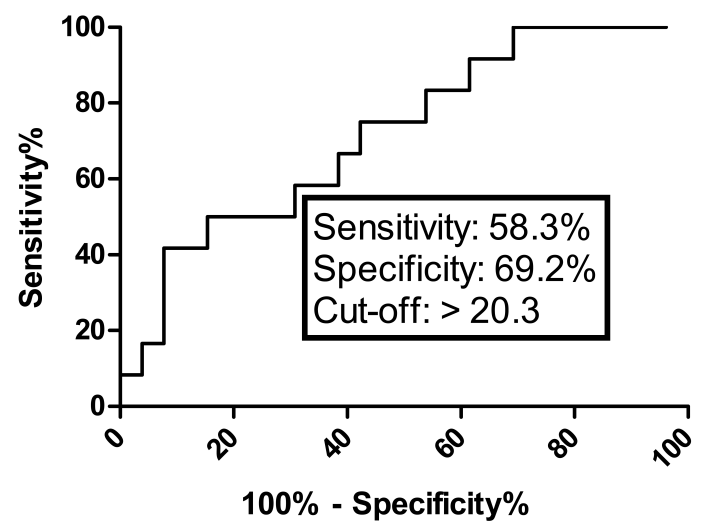

ROC of CD3 MAF MDR at 6wk

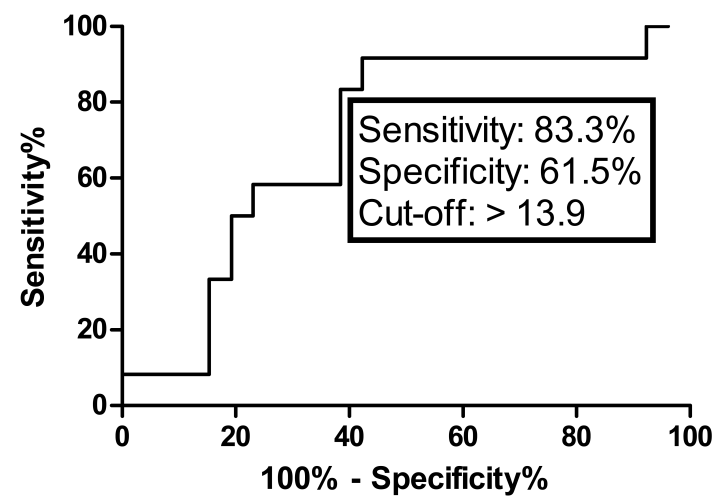

Figure 4. ROC analysis was performed to evaluate the predictive value of MAF for response to treatment in patients with rheumatoid arthritis at the start of biological therapy and at 6 weeks. Patients with MAF values above the respective cutoff thresholds are likely to be nonresponders to treatment. MAFC of CD3 cells at 0 weeks: $\mathrm{p}=0.043$, AUC $=0.68 ;$ MAFC of CD3 cells at 6 weeks: $\mathrm{p}=0.033$, AUC $=0.72 ;$ MAFMDR on CD3 cells at 6 weeks: $\mathrm{p}=0.048, \mathrm{AUC}=0.70$; MAFMRP on CD3 cells at 6 weeks: $p=0.049, \mathrm{AUC}=0.69$. ROC: receiver-operation characteristic curve; MDR: multidrug resistance; MAF: MDR activity factor; MAFC: composite MAF of MRP1 and MDR1; AUC: area under the curve; MRP: MDR protein.

are therefore not substrates of MDR proteins. However, the cytokines they target may indirectly interact with these transporters ${ }^{7,8,9}$. For instance, a recent study described that stimulation with TNF- $\alpha$ induced MDR1 and MRP1 expression through nuclear factor- $\kappa \mathrm{B}$ signaling in astrocytes ${ }^{16}$. Therefore, we hypothesized that MDR activity may be used as a biomarker to predict therapeutic success in RA. A similar crosstalk in lymphocytes could provide the molecular basis of the findings of our clinical study, yet to be confirmed in future investigations.

Our ROC analysis revealed that the assessment of multidrug activity of peripheral blood lymphocytes carries predictive value for response to bDMARD treatment in patients with RA at the start of therapy. Patients with MAF values above the cutoff thresholds are likely to be nonresponders to treatment. Of note, these cutoff values are all below the respective reference ranges in healthy individuals established in our earlier study.
Although baseline MAF values before the start of bDMARD therapy did not differ between healthy controls and the patient and RA groups, such differences were already present between responder and nonresponder patients with RA in the case of T cells. Future studies may be able to explore whether differences in MAF values are present in therapy-naive patients with RA compared to healthy controls, as well as the changes in the activity of the studied transporters over the course of csDMARD treatment until reaching the need for bDMARD therapy. This information could provide more insight into the pathophysiological role of these transporters and may enable even earlier prediction of RA treatment response.

Interestingly, no significant changes of MAF values within the RA patient groups were demonstrated with the progress of treatment (Figure 3). However, considerable intra- and interpatient variations were observed within both patient groups in these values. This observation may be related to

$$
\text { Personal non-commercial use only. The Journal of Rheumatology Copyright @ } 2019 \text {. All rights reserved }
$$


the limitations of our study, such as the relatively small number of patients included in each group and in particular the heterogeneity of the bDMARD treatment received. A larger number of patients in future studies will allow creating and comparing homogeneous patient groups regarding the therapy applied.

Our results indicate that the determination of MAFC values in CD3 cells of patients with RA may be of predictive value prior to the initiation of biological therapy to establish whether the patient will demonstrate sufficient therapeutic response. Measuring MAFC, MAFMRP, and MAFMDR values in CD3 cells at 4 to 6 weeks after the start of treatment further improves the accuracy of prediction of whether adequate therapeutic response may be expected.

\section{REFERENCES}

1. Alamanos Y, Drosos AA. Epidemiology of adult rheumatoid arthritis. Autoimmun Rev 2005;4:130-6.

2. Smolen JS, Landewé R, Bijlsma J, Burmester G, Chatzidionysiou K, Dougados $\mathrm{M}$, et al. EULAR recommendations for the management of rheumatoid arthritis with synthetic and biological disease-modifying antirheumatic drugs: 2016 update. Ann Rheum Dis 2017;76:960-77.

3. Felson DT, Smolen JS, Wells G, Zhang B, van Tuyl LH, Funovits J, et al. American College of Rheumatology/European League against Rheumatism provisional definition of remission in rheumatoid arthritis for clinical trials. Ann Rheum Dis 2011;70:404-13.

4. Verheul MK, Fearon U, Trouw LA, Veale DJ. Biomarkers for rheumatoid and psoriatic arthritis treatments. Clin Immunol 2015;161:2-10

5. Robey RW, Pluchino KM, Hall MD, Fojo AT, Bates SE, Gottesman MM. Revisiting the role of $\mathrm{ABC}$ transporters in multidrug-resistant cancer. Nat Rev Cancer 2018;18:452-64.
6. Márki-Zay J, Tauberné Jakab K, Szerémy P, Krajcsi P. MDR-ABC transporters: biomarkers in rheumatoid arthritis. Clin Exp Rheumatol 2013;31:779-87.

7. García-Carrasco M, Mendoza-Pinto C, Macias Díaz S, Vera-Recabarren M, Vázquez de Lara L, Méndez Martínez S, et al. P-glycoprotein in autoimmune rheumatic diseases. Autoimmun Rev 2015;14:594-600.

8. Ghandadi M, Sahebkar A. Interleukin-6: a critical cytokine in cancer multidrug resistance. Curr Pharm Des 2016;22:518-26.

9. Ronaldson PT, Ashraf T, Bendayan R. Regulation of multidrug resistance protein 1 by tumor necrosis factor alpha in cultured glial cells: involvement of nuclear factor-kappaB and c-Jun N-terminal kinase signaling pathways. Mol Pharmacol 2010;77:644-59.

10. Szerémy P, Tauberné Jakab K, Baráth S, Apjok A, Filkor A, Holló Z, et al. Determination of reference values of MDR-ABC transporter activities in $\mathrm{CD} 3+$ lymphocytes of healthy volunteers using a flow cytometry based method. Cytometry B Clin Cytom 2018 Oct 23 (E-pub ahead of print).

11. Tsujimura S, Saito K, Kohno K, Tanaka Y. Fragmented hyaluronan induces transcriptional up-regulation of the multidrug resistance-1 gene in CD4+ T cells. J Biol Chem 2006;281:38089-97.

12. Tsujimura S, Saito K, Nakayamada S, Nakano K, Tsukada J, Kohno $\mathrm{K}$, et al. Transcriptional regulation of multidrug resistance-1 gene by interleukin-2 in lymphocytes. Genes Cells 2004;9:1265-73.

13. Tsujimura S, Tanaka Y. Disease control by regulation of P-glycoprotein on lymphocytes in patients with rheumatoid arthritis. World J Exp Med 2015;5:225-31.

14. Prasad S, Tripathi D, Rai MK, Aggarwal S, Mittal B, Agarwal V. Multidrug resistance protein-1 expression, function and polymorphisms in patients with rheumatoid arthritis not responding to methotrexate. Int J Rheum Dis 2014;17:878-86.

15. Micsik T, Lớrincz A, Gál J, Schwab R, Peták I. MDR-1 and MRP-1 activity in peripheral blood leukocytes of rheumatoid arthritis patients. Diagn Pathol 2015;10:216.

16. Wang X, Huang S, Jiang Y, Liu Y, Song T, Li D, et al. Reactive astrocytes increase the expression of P-gp and Mrp1 via TNF- $\alpha$ and NF-kB signaling. Mol Med Rep 2018;17:1198-204. 